\title{
Salt and potassium intake among adult Ghanaians: WHO-SAGE Ghana Wave 3
}

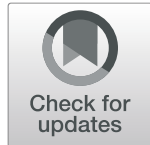

\author{
Elias K. Menyanu', Barbara Corso ${ }^{2}$, Nadia Minicuci $^{2}, \|_{a r i a}$ Rocco ${ }^{2}$, Joanna Russell ${ }^{3}$, Lisa J. Ware ${ }^{4,5}$, Richard Biritwum ${ }^{6}$,
} Paul Kowal ${ }^{7,8}$, Aletta E. Schutte ${ }^{9,10}$ and Karen E. Charlton ${ }^{1,11^{*}}$ (D)

\begin{abstract}
Though Ghana has high hypertension prevalence, the country lacks current national salt consumption data required to build and enhance advocacy for salt reduction. We explored the characteristics of a randomly selected sub sample that had valid urine collection, along with matched survey, anthropometric and BP data $(n=839$, mean age $=60 y$ ), from the World Health Organization's Study on global AGEing and adult health (WHO-SAGE), Ghana Wave $3, n=3053)$. We also investigated the relationship between salt intake and blood pressure (BP) among the cohort. BP was measured in triplicate and $24 \mathrm{~h}$ urine was collected for the determination of urinary sodium ( $\mathrm{Na}$ ), potassium (K), creatinine ( $\mathrm{Cr}$ ) and iodine levels. Hypertension prevalence was $44.3 \%$. Median salt intake was $8.3 \mathrm{~g} /$ day, higher in women compared to men (8.6, interquartile range (IQR) $7.5 \mathrm{~g} /$ day vs $7.5, \mathrm{IQR} 7.4 \mathrm{~g} /$ day, $p<0.01$ ), younger participants (18-49 y) compared to older ones (50+y) (9.7, IQR 7.9 g/day vs 8.1, IQR 7.1 g/day, $p<0.01$ ) and those with higher Body Mass Index (BMI) $\left(>30 \mathrm{~kg} / \mathrm{m}^{2}\right)$ compared to a healthy BMI $\left(18.5-24.9 \mathrm{~kg} / \mathrm{m}^{2}\right)(10.04$, IQR $5.1 \mathrm{~g} /$ day vs 6.2, IQR $5.6 \mathrm{~g} /$ day, $p<0.01)$. More than three quarters $(77 \%, n=647)$ of participants had salt intakes above the WHO maximum recommendation of $5 \mathrm{~g} / \mathrm{d}$, and nearly two thirds $(65 \%, n=548)$ had daily $\mathrm{K}$ intakes below the recommended level of $90 \mathrm{mmol}$. Dietary sodium to potassium ( $\mathrm{Na}: \mathrm{K}$ ) ratios above $2 \mathrm{mmol} / \mathrm{mmol}$ were positively associated with increasing BP with age. Population-based interventions to reduce salt intake and increase K consumption are needed.
\end{abstract}

Keywords: Hypertension, Salt intake, Potassium, 24 h urine, Ghana

\section{Introduction}

Cardiovascular diseases (CVDs) remain a major contributory factor for mortality and morbidity worldwide, accounting for 17.9 million deaths in 2016, and representing a third of all deaths globally [1]. While Lowand Middle-Income Countries (LMICs) already share the greatest impact of CVD-related deaths, with rates of 300-600 deaths per 100,000 population, these are predicted to increase, causing premature, avoidable loss of lives [2]. Hypertension is a major risk factor for CVD

\footnotetext{
* Correspondence: karenc@uow.edu.au

'School of Medicine, Faculty of Science, Medicine and Health, University of Wollongong, Northfields Avenue, Wollongong, NSW 2522, Australia

${ }^{11}$ Illawarra Health and Medical Research Institute, Wollongong, NSW 2522, Australia

Full list of author information is available at the end of the article
}

and accounted for more than $50 \%$ of CVD-specific deaths in 2012 [3]. It has been estimated that the burden of hypertension will increase to approximately 1.56 billion people globally by 2025 , with larger populations of hypertensives living in LMICs [3-5].

Hypertension is a common condition in Ghana, where its prevalence has increased more than two-fold over just two decades (1988-2007) [6]. A recent nationally representative study among participants largely 50 years and older, in Ghana, reported the prevalence of hypertension to be $58.9 \%$ in adults with about one-fifth of those with hypertension being aware of having the condition [7]]. Reducing population-level prevalence of hypertension by $30 \%$ by the year 2030 is identified by WHO as one of the nine voluntary global targets to reduce non communicable

(c) The Author(s). 2020 Open Access This article is licensed under a Creative Commons Attribution 4.0 International License, which permits use, sharing, adaptation, distribution and reproduction in any medium or format, as long as you give appropriate credit to the original author(s) and the source, provide a link to the Creative Commons licence, and indicate if changes were made. The images or other third party material in this article are included in the article's Creative Commons licence, unless indicated otherwise in a credit line to the material. If material is not included in the article's Creative Commons licence and your intended use is not permitted by statutory regulation or exceeds the permitted use, you will need to obtain permission directly from the copyright holder. To view a copy of this licence, visit http://creativecommons.org/licenses/by/4.0/. The Creative Commons Public Domain Dedication waiver (http://creativecommons.org/publicdomain/zero/1.0/) applies to the data made available in this article, unless otherwise stated in a credit line to the data. 
diseases (NCDs) [8]. Salt reduction has been identified by the WHO as one 'best buys' approach to reduce overall CVD risk through lowering of (BP) [9].

Increased consumption of salt $(>2 \mathrm{~g} \mathrm{Na}$ /day, equivalent to $5 \mathrm{~g}$ salt/day) together with insufficient potassium (K) intake $(<3.5 \mathrm{~g} /$ day $)$ are strongly associated with a number of NCDs including hypertension [10-14]. Several systematic literature reviews, meta analyses and randomized-controlled trials have shown that reduced sodium $(\mathrm{Na})$ consumption results in a decline in blood pressure in both hypertensives and normotensive adults [12, 14-17], which saves lives [18]. In view of this, 'WHO's SHAKE the Salt Habit' resource has emphasized the importance of measuring and monitoring population salt consumption patterns in order to inform stakeholders about strategies to reduce salt intake and evaluate the effect of any implemented salt reduction programmes [19]. Though Ghana is committed to the World Health Assembly's target of $30 \%$ relative reduction in the population salt intake by 2025 [10], the country has made slow progress towards achieving the target due to other competing public health priorities and limited resources [20].

Few studies have been conducted to assess salt intake in the Ghanaian population [21]. A lack of recent, nationally representative data on population salt consumption among adults has further been a disincentive for advocacy around salt reduction efforts [22]. The current study was undertaken to obtain a reliable estimate of population salt intake and salt use behaviour, and to assess the relationship between salt and $\mathrm{K}$ intake with blood pressure (BP) in Ghanaian adults.

\section{Methods}

\section{Study design, population and outcomes}

This study is a nested sub study of WHO's Study on global AGEing and adult health (WHO - SAGE) Wave 3 Ghana, which collected $24 \mathrm{~h}$ urine samples for analysis of $\mathrm{Na}$ and $\mathrm{K}$ [23]. WHO - SAGE is a longitudinal cohort study conducted in six LMICs (China, Ghana, India, Russia, Mexico and South Africa) with the aim to examine the ageing process and address health inequalities among adult populations [24]. Participants were sampled based on a design used in the 2003 World Health Survey with primary sampling units (households) stratified by region and location (urban/rural) The study design randomly selects 24 households from an enumeration area (twenty 50+ year households and four 18-49 year households). In the 50+ year households, all participants are selected and compares with a smaller number of participants selected from the 18-49 year households, details of which are described elsewhere [25]. WHO SAGE Ghana Wave 3 data collection was completed over a period of 1 year (July 2018-June 2019) with $n=110024 \mathrm{~h}$ urine samples collected nationwide. Out of 5570 participants recruited for WHO - SAGE Ghana Wave 3, 1102 (20\%), were randomly selected to provide urine samples (nested sub study). Of these, 76.7\% $(n=844)$ had successful (BP) data and $88.6 \%(n=976)$ had complete urine samples. However, fewer participants $(n=839)$ had a complete urine collection, accompanied with survey, anthropometric and valid BP data (Fig. 1).

Field worker teams consisted of 3-5 interviewers per team who visited participants in their homes and workplaces to conduct interviews. All field workers were trained by the central WHO SAGE team with standardized survey materials [24]. Surveys were conducted in the participants' preferred home language with the use of the computer assisted personal interview (CAPI) method.

The WHO/Pan American Health Organization (PAHO) protocol was utilised in the determination and collection of $\mathrm{Na}, \mathrm{K}$ and $\mathrm{Cr}$ in the $24 \mathrm{~h}$ urine collections. In collecting urine samples, 5-l urine bottles containing $1 \mathrm{~g}$ thymol as preservative, were given to participants to collect $24 \mathrm{~h}$ urine. The collection procedure was thoroughly explained to participants. In brief it is as follows: 1) void the 'first urine' and note the time but include the 'last urine' 2) keep the bottles to yourself and collect only your own urine 3 ) collect all urine passed within the $24 \mathrm{~h}$ and 4) keep collected urine in a cool place [26]. The $24 \mathrm{~h}$ urine was collected, thoroughly mixed, volumes recorded, 3 aliquots of $5 \mathrm{ml}$ were generated and samples kept in cold boxes and transported to the Noguchi Memorial Institute for Medical Research of the University of the Ghana (NMIMR-UG). Urine samples were considered valid if the volume was $\geq 300 \mathrm{ml}[27,28]$. Additionally, iodine was assessed for analysis because Ghana had a universal iodine programme in place.

Analysis were undertaken by NMIMR-UG, that followed the WHO/PAHO protocol for quantitative analysis of $\mathrm{Na}, \mathrm{K}, \mathrm{Cr}$ in urine samples [26]. Urine samples for iodine analyses were stored at $-20^{\circ}$ and batch analysed using the Sandell-Kolthoff method with ammonium persulfate digestion and microplate [29]. Creatinine excretion in this population was much lower than that reported for many other populations, from which $\mathrm{Cr}$ cut-off values have been determined to indicate completeness of $24 \mathrm{~h}$ urine collection [28]. Because of this, only urine volume was used to indicate whether urine samples were considered to represent completeness. This approach has been used in other multi-country studies including; the International Population Study on Macronutrients and Blood Pressure [INTERMAP] and the International Study of Sodium, Potassium, and Blood Pressure [INTERSALT] [27]. In the first enumerator areas (EAs) sampled within the Accra region, some calculated salt values were unfeasibly high ( $>40 \mathrm{~g} / \mathrm{d}, n=40$,) due to unacceptable storage conditions [30]. A follow-up validation study 


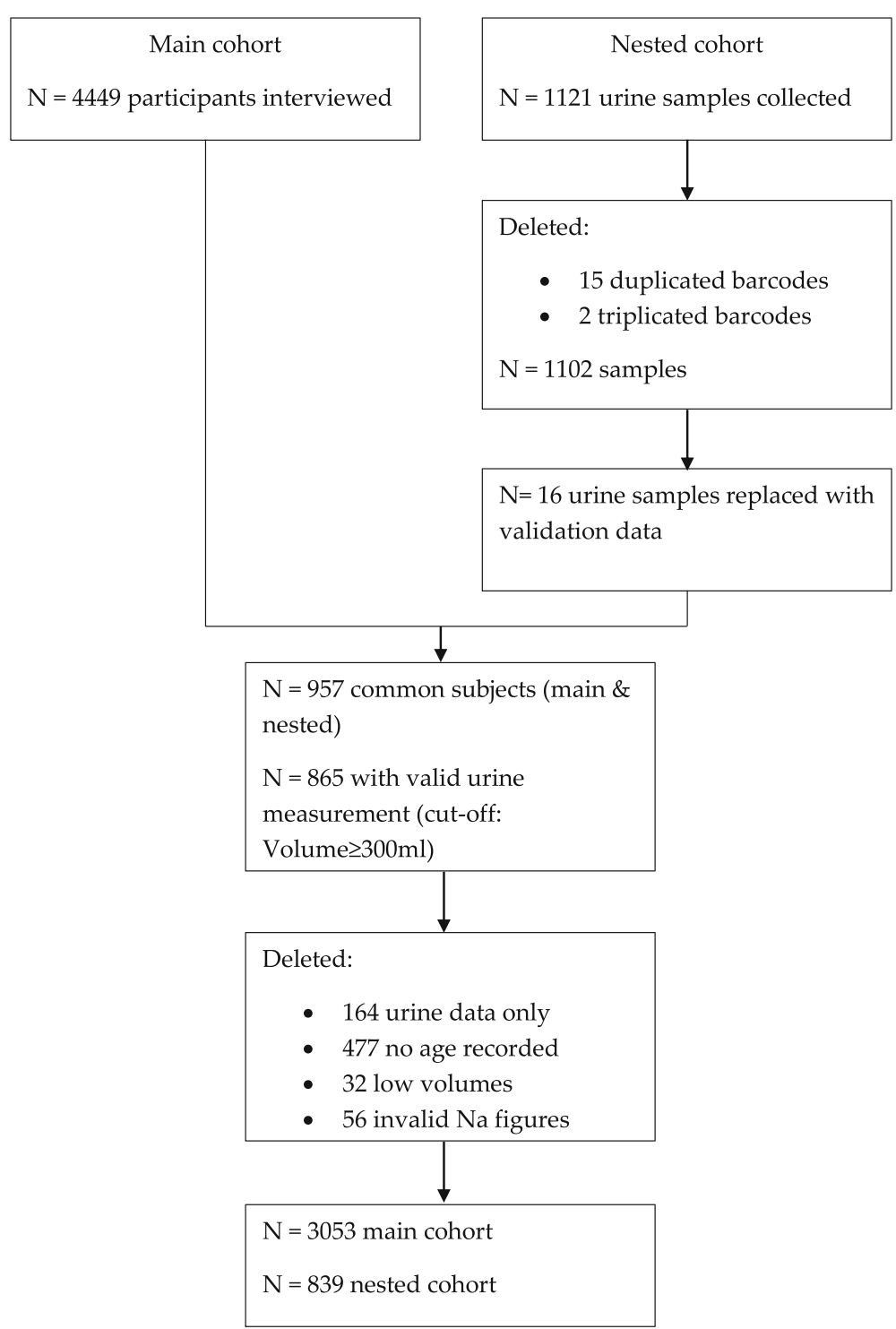

Fig. 1 Study flow chart

conducted in $n=67$ participants included the collection of repeated $24 \mathrm{~h}$ urine samples from the same participants in one EA (Alajo; $n=19$ ), while an additional two non-urine EAs from the larger survey sample were visited as a comparator (Mataheko; $n=$ 24 and Nima; $n=24$ ). A second laboratory (Liberty Medical Laboratory, Accra) also performed duplicate analyses in the validation study. Values for Dosise EA $(n=23)$ were excluded after the validation study because they were confirmed as being unfeasible, and the validation study values replaced original measures for the case of Alajo $(n=16 ; 3$ barcodes of urine samples in validation study were excluded because they could not be matched with those of the original study) (Supplementary Table 1).
Sodium $(\mathrm{mmol} / \mathrm{l})$ in the $24 \mathrm{~h}$ urine sample was converted to salt $(\mathrm{g} / \mathrm{d})$ using the formula: $\mathrm{Na} \mathrm{mmol} / \mathrm{l} \times 24 \mathrm{~h}$ volume (litres) $\times 23.1$ (molecular weight of $\mathrm{Na}) / 390(390$ $\mathrm{mg} \mathrm{Na}$ per $1 \mathrm{~g} \mathrm{Nacl}$ (salt). From this variable, participants were categorised as having low $(<5 \mathrm{~g} /$ day $)$, medium (5-9g/day) or high ( $>9 \mathrm{~g} /$ day) salt intake. $\mathrm{K}$ $(\mathrm{mmol} / \mathrm{l})$ in the $24 \mathrm{~h}$ urine sample was converted to $\mathrm{K}$ $(\mathrm{mmol} / \mathrm{d})$ using the formula: $\mathrm{K}(\mathrm{mmol} / \mathrm{l}) \times 24 \mathrm{~h}$ volume (litres) [31]. The ratio $\mathrm{Na}(\mathrm{mmol} / \mathrm{L})$ to $\mathrm{K}(\mathrm{mmol} / \mathrm{L})$ was created (Na:K) and categorised as low $(<2)$, medium $(2-5)$ or high ( $>5$ ). BP was measured by field workers with wrist worn BP devices with positional sensors (Omron R6, Japan) [32] that have been validated by the European Society of Hypertension International Protocol [32-34]. After being seated with legs uncrossed for $5 \mathrm{~min}$, three BP 
readings were taken on the left wrist ( 1 min between each measurement) while the wrist was placed precisely at the level of heart. The last two readings were averaged as a measure of the participant's BP. Hypertension classification was based on the European Society for Hypertension Guidelines (2018) which defines hypertension as systolic $\geq 140$ and/or diastolic $\geq 90 \mathrm{mmHg}$ [35]. BP readings in the database were determined valid if: Systolic $(\mathrm{SBP})>$ Diastolic (DBP); and SBP was between 80 and $270 \mathrm{mmHg}$; and DBP was between 40 and $180 \mathrm{mmHg}$; and SBP minus DBP (Pulse Pressure, PP) $>13 \mathrm{mmHg}$. Mean Arterial Pressure (MAP) is the average arterial pressure throughout one cardiac cycle, systole, and diastole. MAP was calculated using the formulae: $\mathrm{MAP}=\mathrm{SBP}+2(\mathrm{DBP}) / 3$ [36]. Hypertension status was measured as self-reported treatment or having a measured $\mathrm{BP} \geq 140 / 90 \mathrm{mmHg}$. Hypertension awareness was based on self-reported previous diagnosis of hypertension in those with $\mathrm{BP} \geq 140 / 90$ $\mathrm{mmHg}$. Treatment was determined from self-reported medication use for hypertension within the last 2 weeks. Hypertension control was determined by self-reported medication use within the last 2 weeks and a measured BP less than $140 / 90 \mathrm{mmHg}$. Individuals with no current medication use and a measured BP $<140 / 90 \mathrm{mmHg}$ were categorized as non-hypertensive.

Weight was measured with calibrated scales, while height was measured with a portable stadiometer. Data were also collected on health indicators such as tobacco, alcohol use, salt use behaviours and previous disease conditions. Physical activity levels were measured using the Global Physical Activity Questionnaire [37]. For salt use behaviour questions, responses such as 'always' and 'often' were combined to represent 'frequent use', whilst 'sometimes' and 'rarely' were combined to represent 'infrequent use'.

Prior to taking part in the study, study measures were explained to participants in their home languages and written informed consent was obtained. The study complied with the Declaration of Helsinki with ethical approval from the WHO Ethics Committee (RPC 149) and the University of Ghana Medical School Ethics and Protocol Review Committee (MS-Et/M.03 - P 3.1/20052006).

\section{Statistical analysis}

Data were analysed using Stata Statistical Software: Release 15 (Stata Corp LLC, 2017; College Station, USA). Data distribution was checked visually and with the Shapiro Wilks test for normality. Descriptive statistics of frequencies, percentages and median (interquartile range, IQR) were used to describe participants' characteristics. Categorical variables were evaluated using frequencies, Pearson's ChiSquare and Fisher's Exact tests. Comparison between groups for non-parametric data was examined using
Mann-Whitney U and Kruskal-Wallis tests and Spearman's Rho was used to test for correlations. Linear regressions were used to evaluate the associations between variables. To test regression slopes for equality between the three salt level groups and between the three Na:K ratio groups, the interaction terms of groups with age was added into the models. To test if a difference in the group's coefficients (i.e. slope) was present, the $\mathrm{F}$ test was applied. If significant, post-estimation was pursued to identify which group differed from the others.

\section{Results}

Table 1 compares sociodemographic characteristics of the nested sub study sample with those included in the total Wave 3 survey collection.

Hypertension prevalence in the nested sub study was $44.3 \%(n=370)$ with 41.4 and $46.1 \%$ for men and women respectively. Of those with hypertension, 59.7\% were aware of their condition $(n=222)$ of which $69.1 \%$ ( $n=154$ ) were receiving prescribed antihypertensive medication. Of those being treated for hypertension, $51 \%(n=77)$ had controlled BP (ie BP $\leq 140 / 90)$.

Among participants with both urine, survey data and BP data $(n=839)$, median intake of salt was equivalent to $8.3 \mathrm{~g} /$ day (IQR 7.5) (mean $=10.2 \mathrm{~g} \pm 7.2$ ). Salt intake was higher in: (1) women compared to men; median 8.6 (7.5)g/day vs 7.5 (7.4)g/day, $p<0.01$; (2) younger participants $(18-49$ y) compared to older ones $(50+y)$; median 9.7 (7.9)g/day vs 8.1 (7.1)g/day, $p<0.01$; and (3) those with higher BMI $\left(>30 \mathrm{~kg} / \mathrm{m}^{2}\right)$ compared to a desirable BMI $\left(18.5-24.9 \mathrm{~kg} / \mathrm{m}^{2}\right)$; median $10.04(5.1) \mathrm{g} /$ day vs 6.2 (5.6)g/day, $p<0.01$. There was no significant difference in salt consumption between rural and urban dwellers. Overall, 77.7\% $(n=647)$ of participants had salt intakes above the WHO maximum recommendation of $5 \mathrm{~g} / \mathrm{d}$, with $38.9 \%$ consuming more than twice this level $(>10 \mathrm{~g} /$ $\mathrm{d}, n=321$ ) and $16.3 \%$ consuming more than three times the recommended level $(>15 \mathrm{~g} / \mathrm{d}, n=137)$. More men than women $(27.1 \%(n=73)$ vs $19.9 \%(n=122) ; p=0.02)$ and older participants than younger ones $(24.8 \%(n=164)$ vs $12.1 \%(n=21) ; p<0.01)$ achieved the WHO' salt recommendation of $\leq 5 \mathrm{~g}$ salt/day (Supplementary Tables 2 and 3).

According to groupings by salt intake (low, $<5 \mathrm{~g} / \mathrm{d}$; medium, 5-9 g/d; high, $>9 \mathrm{~g} / \mathrm{d}$ ), those with higher salt intakes were significantly younger, had higher BMI, a higher urinary $\mathrm{K}$ excretion, a higher urinary $\mathrm{Na}$ : $\mathrm{K}$ and lower urinary iodine concentrations. However, BP and hypertension status did not differ between the groups (Table 2).

In response to the salt behaviour questions (Supplementary Table 4), $12.8 \%(n=107)$ of participants reported that they frequently (always and often) added salt to food at the table with significantly greater numbers of younger $(27.3 \%, n=28$, 
Table 1 Demographic profile of SAGE GHA W3 survey participants and subsample with valid urine data (urine data considered valid if volume $\geq 300 \mathrm{ml}$ )

\begin{tabular}{|c|c|c|c|}
\hline & Main SAGE cohort $(n=3053)$ & Salt sub study sample $(n=839)$ & $P$-value \\
\hline \multicolumn{4}{|l|}{ Age } \\
\hline Years & $60(20)$ & $60(19)$ & 0.3294 \\
\hline $18-49$ years, n (\%) & $717(23.5)$ & $174(20.7)$ & 0.0100 \\
\hline 50 plus years, $\mathrm{n}(\%)$ & $2336(76.5)$ & $665(79.3)$ & 0.4494 \\
\hline $\operatorname{Sex}, n(\%)$ & & & 0.001 \\
\hline Men & $1183(38.8)$ & $371(32.3)$ & \\
\hline Women & $1870(61.2)$ & $568(67.7)$ & \\
\hline Ethnicity, n (\%) & & & $<0.0001$ \\
\hline Akan & $1544(50.6)$ & $497(59.2)$ & \\
\hline Ewe & $216(7.1)$ & $58(6.9)$ & \\
\hline Ga-Adangbe & $224(7.3)$ & $45(5.4)$ & \\
\hline Gruma & $30(1.0)$ & $6(0.7)$ & \\
\hline Grusi & $76(2.5)$ & $12(1.4)$ & \\
\hline Guan & $38(1.2)$ & $16(1.9)$ & \\
\hline Mande-Busanga & $63(2.1)$ & $3(0.4)$ & \\
\hline Mole-Dagbon & $174(5.7)$ & $24(2.9)$ & \\
\hline Other & $612(20.0)$ & $174(20.7)$ & \\
\hline Residence, $n(\%)$ & & & $<0.0001$ \\
\hline Urban & $1301(42.6)$ & $457(54.5)$ & \\
\hline Rural & $1752(57.4)$ & $382(45.5)$ & \\
\hline Marital status, $n(\%)$ & & & 0.002 \\
\hline Never married & $208(6.8)$ & $39(4.6)$ & \\
\hline Married/cohabiting & $1760(57.7)$ & $470(56.0)$ & \\
\hline Separate/divorced & $369(12.1)$ & $87(10.4)$ & \\
\hline Widowed & $715(23.4)$ & $243(29.0)$ & \\
\hline Never been to school, $n(\%)$ & $1119(36.7)$ & $240(28.6)$ & $<0.0001$ \\
\hline Education (years) & $10(5)$ & $10(5)$ & 0.0139 \\
\hline$B M I \mathrm{~kg} / \mathrm{m}^{2}$ & $24.2(6.8)$ & $25.4(7.4)$ & $<0.01$ \\
\hline Waist to height ratio & $0.54(0.11)$ & $0.56(0.13)$ & $<0.01$ \\
\hline Never used alcohol, $n(\%)$ & $1865(61.3)$ & $483(57.6)$ & 0.052 \\
\hline Never used tobacco, $n(\%)$ & $2739(90.0)$ & $759(90.5)$ & 0.68 \\
\hline Systolic BP ( $m m$ Hg) & $124(26.5)$ & $126.5(29)$ & $<0.01$ \\
\hline Diastolic BP (mm Hg) & $76(17)$ & $76.5(17)$ & 0.83 \\
\hline Mean Arterial Pressure (Map) $\mathrm{mmHG}$ & $92.5(19.2)$ & $93.2(20.7)$ & 0.24 \\
\hline Hypertension, n(\%) & $1132(37.6)$ & $370(44.3)$ & $<0.01$ \\
\hline Diabetes, $n(\%)$ & $157(5.2)$ & $67(8.0)$ & 0.01 \\
\hline Portions of fruit, $n(\%)$ & & & 0.05 \\
\hline $\begin{array}{l}\text { Met WHO's recommendation of at least } 5 \text { servings } \\
\text { of friuts and vegetables }\end{array}$ & $1591(57.4)$ & $442(54.5)$ & 0.14 \\
\hline Frequently add salt to food at table, $n(\%)$ & $642(21.1)$ & $107(12.7)$ & $<0.01$ \\
\hline Frequently add salt to food during cooking, $n(\%)$ & $2527(83.0)$ & $672(80.1)$ & 0.05 \\
\hline Believe they consume too much salt, $n(\%)$ & $265(8.8)$ & $92(11.0)$ & 0.05 \\
\hline Believe a high salt diet is bad for health, $n(\%)$ & $2247(75.9)$ & $651(80.0)$ & 0.02 \\
\hline Regularly control of salt intake, n(\%) & $1243(42.1)$ & $394(48.5)$ & $<0.01$ \\
\hline
\end{tabular}

Nested sub sample: all respondent with CAPI data and valid urine, sex and age recorded. Some variables may contain missing data. Data are presented as median (IRQ) unless otherwise indicated. Hypertensive by measured $B P \geq 140$ and/or $90 \mathrm{mmHg}$ or previous diagnosis (self-reported). Ethnicity, marital status, education, alcohol/tobacco use and diabetes prevalence by self-report. Mann-Whitney Test used to compare medians, Pearson Chi-Square test and Fisher's Exact Test used to compare proportional data 
Table 2 Nested cohort by salt excretion levels, low, medium and high salt groups, WHO - SAGE Ghana Wave 3 (2019)

\begin{tabular}{|c|c|c|c|c|}
\hline Characteristics & $\begin{array}{l}\text { Low salt } \\
<5 \mathrm{~g} / \mathrm{d} \\
(n=186)\end{array}$ & $\begin{array}{l}\text { Medium salt } \\
5-9 \mathrm{~g} / \mathrm{d} \\
(n=269)\end{array}$ & $\begin{array}{l}\text { High salt } \\
>9 \mathrm{~g} / \mathrm{d} \\
(n=378)\end{array}$ & $p$ value \\
\hline Age yrs & $64(20)$ & $60(17)$ & $58(18)$ & $<0.01$ \\
\hline Sex male, $n(\%)$ & $\begin{array}{l}n=185 \\
73(39.5)\end{array}$ & $\begin{array}{l}n=269 \\
90(33.5)\end{array}$ & $\begin{array}{l}n=378 \\
105(27.8)\end{array}$ & 0.02 \\
\hline Ethnicity n (\%) & $n=184$ & $n=268$ & $n=376$ & 0.03 \\
\hline Akan & $122(66.3)$ & $168(62.7)$ & $203(54.0)$ & \\
\hline Ga & $7(3.8)$ & $11(4.1)$ & $27(7.2)$ & \\
\hline Ewe & $14(7.6)$ & $18(6.7)$ & $24(6.4)$ & \\
\hline Mole-dagbon & $5(2.7)$ & $6(2.2)$ & $13(3.5)$ & \\
\hline Mole-busanga & $1(0.5)$ & $0(0.0)$ & $2(0.5)$ & \\
\hline Grusi & $3(1.6)$ & $3(1.1)$ & $6(1.6)$ & \\
\hline Guan & $0(0.0 \%)$ & $11(4.1 \%)$ & $5(1.3)$ & \\
\hline Gruma & $1(0.5)$ & $3(1.1)$ & $2(0.5)$ & \\
\hline Other & $31(16.8)$ & 48 (17.9) & $94(25.0)$ & \\
\hline Urban n (\%) & $\begin{array}{l}n=185 \\
102(55.1)\end{array}$ & $\begin{array}{l}n=269 \\
149(55.4)\end{array}$ & $\begin{array}{l}n=378 \\
200(52.9)\end{array}$ & 0.79 \\
\hline Education (years) & $\begin{array}{l}n=118 \\
10(5)\end{array}$ & $\begin{array}{l}n=179 \\
10(3)\end{array}$ & $\begin{array}{l}n=250 \\
10(5)\end{array}$ & 0.07 \\
\hline$B M l, \mathrm{~kg} / \mathrm{m}^{2}$ & $\begin{array}{l}n=179 \\
23.8(6.5)^{\mathrm{a}}\end{array}$ & $\begin{array}{l}n=258 \\
25.5(7.8)\end{array}$ & $\begin{array}{l}n=356 \\
26.2(7.5)^{\mathrm{a}}\end{array}$ & $<0.01$ \\
\hline Salt intake $\mathrm{g} / \mathrm{d}$ & $\begin{array}{l}n=186 \\
3.6(1.5)^{\mathrm{ab}}\end{array}$ & $\begin{array}{l}n=269 \\
6.7(2.0)^{\mathrm{ac}}\end{array}$ & $\begin{array}{l}n=371 \\
13.4(8.9)^{b c}\end{array}$ & $<0.01$ \\
\hline K intake, mmol/d & $\begin{array}{l}n=186 \\
32.0(25.8)^{\mathrm{ab}}\end{array}$ & $\begin{array}{l}n=269 \\
54.9(43.8)^{\mathrm{ac}}\end{array}$ & $\begin{array}{l}n=378 \\
112.3(115.8)^{b c}\end{array}$ & $<0.01$ \\
\hline $\mathrm{Na}: \mathrm{K}$ & $\begin{array}{l}n=186 \\
1.7(1.2)^{\mathrm{ab}}\end{array}$ & $\begin{array}{l}n=269 \\
2.0(1.4)^{\mathrm{ac}}\end{array}$ & $\begin{array}{l}n=378 \\
2.3(1.9)^{b c}\end{array}$ & $<0.01$ \\
\hline Urinary iodine excretion, mmol/day & $\begin{array}{l}n=150 \\
166.2(168.5)^{b}\end{array}$ & $\begin{array}{l}n=211 \\
148.2(131.4)^{a}\end{array}$ & $\begin{array}{l}n=292 \\
117.6(132.8)^{\mathrm{ab}}\end{array}$ & $<0.01$ \\
\hline Systolic $B P, \mathrm{mmHg}$ & $\begin{array}{l}n=186 \\
129(31.0)\end{array}$ & $\begin{array}{l}n=266 \\
126(31.0)\end{array}$ & $\begin{array}{l}n=375 \\
126.5(29.0)\end{array}$ & 0.73 \\
\hline Diastolic $B P, \mathrm{mmHg}$ & $\begin{array}{l}n=188 \\
76(18.0)\end{array}$ & $\begin{array}{l}n=270 \\
75.5(17)\end{array}$ & $\begin{array}{l}n=379 \\
77.5(117.0)\end{array}$ & 0.68 \\
\hline Pulse pressure, $\mathrm{mmHg}$ & $\begin{array}{l}n=186 \\
50.5(16.7)\end{array}$ & $\begin{array}{l}n=266 \\
49.5(17.6)\end{array}$ & $\begin{array}{l}n=375 \\
49.5(16)\end{array}$ & 0.20 \\
\hline Mean Arterial Pressure (MAP), $\mathrm{mmHg}$ & $\begin{array}{l}n=185 \\
94.2(21.3)\end{array}$ & $\begin{array}{l}n=266 \\
110(41.0)\end{array}$ & $\begin{array}{l}n=376 \\
93.5(19.4)\end{array}$ & 0.85 \\
\hline Hypertension Prevalence, n (\%) & $\begin{array}{l}n=186 \\
94(50.5)\end{array}$ & $\begin{array}{l}n=268 \\
110(41.0)\end{array}$ & $\begin{array}{l}n=378 \\
166(44.3)\end{array}$ & 0.13 \\
\hline Hypertension awareness, n (\% of hypertension prevalence) & $\begin{array}{l}n=94 \\
51(54.8)\end{array}$ & $\begin{array}{l}n=110 \\
62(55.4)\end{array}$ & $\begin{array}{l}n=166 \\
108(65.1)\end{array}$ & 0.16 \\
\hline $\begin{array}{l}\text { Hypertension medication, current use } n \\
\text { (\% of hypertension awareness) }\end{array}$ & $\begin{array}{l}n=51 \\
31(60.8)\end{array}$ & $\begin{array}{l}n=62 \\
47(75.8)\end{array}$ & $\begin{array}{l}n=108 \\
74(68.5)\end{array}$ & 0.23 \\
\hline Hypertension control, n (\% of Hypertension medication) & $\begin{array}{l}n=31 \\
16(51.6)\end{array}$ & $\begin{array}{l}n=47 \\
22(46.8)\end{array}$ & $\begin{array}{l}n=74 \\
39(52.7)\end{array}$ & 0.82 \\
\hline Stroke, $n(\%)$ & $\begin{array}{l}n=185 \\
6(3.2)\end{array}$ & $\begin{array}{l}n=269 \\
6(2.2)\end{array}$ & $\begin{array}{l}n=378 \\
6(1.6)\end{array}$ & 0.44 \\
\hline Diabetes, $n$ (\%) & $\begin{array}{l}n=185 \\
10(5.4)\end{array}$ & $\begin{array}{l}n=269 \\
24(8.9)\end{array}$ & $\begin{array}{l}n=378 \\
32(8.5)\end{array}$ & 0.37 \\
\hline Tobacco use, n (\%) & $\begin{array}{l}n=182 \\
25(13.7)\end{array}$ & $\begin{array}{l}n=269 \\
25(9.3)\end{array}$ & $\begin{array}{l}n=378 \\
29(7.7)\end{array}$ & 0.72 \\
\hline Alcohol use, $n(\%)$ & $n=182$ & $n=269$ & $n=378$ & 0.82 \\
\hline
\end{tabular}


Table 2 Nested cohort by salt excretion levels, low, medium and high salt groups, WHO - SAGE Ghana Wave 3 (2019) (Continued)

\begin{tabular}{|c|c|c|c|c|}
\hline Characteristics & $\begin{array}{l}\text { Low salt } \\
<5 \mathrm{~g} / \mathrm{d} \\
(n=186)\end{array}$ & $\begin{array}{l}\text { Medium salt } \\
5-9 \mathrm{~g} / \mathrm{d} \\
(n=269)\end{array}$ & $\begin{array}{l}\text { High salt } \\
>9 \mathrm{~g} / \mathrm{d} \\
(n=378)\end{array}$ & $p$ value \\
\hline & $80(44.0)$ & $115(42.8)$ & $156(41.3)$ & \\
\hline Fruits and Veg., met recommendation $n(\%)$ & $\begin{array}{l}n=186 \\
97(52.2)\end{array}$ & $\begin{array}{l}n=269 \\
152(56.5)\end{array}$ & $\begin{array}{l}n=378 \\
193(51.1)\end{array}$ & 0.38 \\
\hline Frequently adds salt to food at the table, $n(\%)$ & $\begin{array}{l}n=158 \\
24(13)\end{array}$ & $\begin{array}{l}n=269 \\
35(13)\end{array}$ & $\begin{array}{l}n=279 \\
(12.4)\end{array}$ & 0.65 \\
\hline Frequently adds salt to cooking at home, $n$ (\%) & $\begin{array}{l}n=185 \\
149(80.5)\end{array}$ & $\begin{array}{l}n=296 \\
225(83.6)\end{array}$ & $\begin{array}{l}n=378 \\
291(77)\end{array}$ & 0.05 \\
\hline Believe that they are eating just the right amount of salt $n(\%)$ & $\begin{array}{l}n=185 \\
105(56.8)\end{array}$ & $\begin{array}{l}n=267 \\
169(63.3)\end{array}$ & $\begin{array}{l}n=376 \\
228(60.6)\end{array}$ & 0.18 \\
\hline Believe a high salt diet can cause a serious health problem, $n(\%)$ & $\begin{array}{l}n=180 \\
144(80.0)\end{array}$ & $\begin{array}{l}n=259 \\
207(79.9)\end{array}$ & $\begin{array}{l}n=368 \\
296(80.4)\end{array}$ & 0.99 \\
\hline Does something to control salt consumption, $n$ (\%) & $\begin{array}{l}n=183 \\
82(44.8)\end{array}$ & $\begin{array}{l}n=260 \\
126(48.5)\end{array}$ & $\begin{array}{l}n=363 \\
182(50.1)\end{array}$ & 0.50 \\
\hline
\end{tabular}

Data are presented as median (IQR, interquartile range) unless otherwise stated. BMI, body; hypertension prevalence, BP $\geq 140 / 90$ or previous diagnosis; Current use of hypertension medication represents current use in the last 2 weeks; BMI, body mass index; tobacco use by self-report; alcohol use by self-report; met fruits and vegetable recommendation indicates consumed five or more servings of fruits and vegetables a day; salt behaviour responses, frequently indicates 'always' and 'often'; continuous variables compared using Independent Kruskal-Wallis Test and categorical variables compared using Pearson Chi-Square Test and Fisher's Exact Test. a, b, c; sig at $p<0.05$

$p=0.02)$ and rural $(61 \%, n=61,<0.01)$ dwellers responding affirmatively to this question. More than three quarters $(80.1 \%, n=671)$ of the surveyed population reported that they frequently added salt to food at home during cooking while $60.9 \%(n=508)$ perceived themselves to consume the right amount of salt. While 19.9\% $(n=126)$ did not know that a high salt diet could cause a serious health problem, $48.5 \%$, $(n=394)$ of the participants reported actively doing something on a regular basis to control their salt intake. Of those who knew a high salt diet could cause a serious health problem, $58.9 \%(n=378)$ did something on a regular basis to control their salt intake.

Almost two-thirds $(65 \%, n=548)$ of participants did not meet the daily $\mathrm{K}$ recommendation of $\geq 90 \mathrm{mmol}$ [38], with those in the lowest salt intake group having the lowest urinary concentrations. While $42.6 \%(n=141)$ of urban participants met the guidelines for $\mathrm{K}$ intake, this was the case for only $28.6 \%(n=101)$ of participants residing in rural areas $(p<0.01)$. Only $7.6 \%(n=63)$ achieved the recommended $\mathrm{Na}$ : $\mathrm{K}$ ratio of $\leq 1 \mathrm{mmol}$ [38]. There was a strong positive correlation between $24 \mathrm{~h}$ urinary $\mathrm{Na}$ and $\mathrm{K}$ concentrations (Spearman's rho $=0.71$, $p<0.01)$. Linear regression indicated that urinary $\mathrm{Na}$ excretion accounted for more than half the variability in urinary $\mathrm{K}$ excretion $\left(R^{2}=0.51, \mathrm{~F}(1,832)=861, p=<\right.$ $0.01)$. Urinary $\mathrm{K}$ excretion increased by $0.71 \mathrm{mmol} /$ day for each $\mathrm{mmol} /$ day increase in urinary $\mathrm{Na}$ such that $\mathrm{Na}$ : $\mathrm{K}$ ratio was significantly higher in the high salt group compared to the low salt group (median 2.3 (1.9)g/day vs $1.7(1.9) \mathrm{g} /$ day, respectively, $p<0.01)$.

The high Na: K group had a significantly higher BMI than those in the lowest Na: K group (median 28.1, IQR 8.2 vs 24.8 , IQR $7.0, p=0.01$ ) and had higher number of participants who reported doing something on regular basis to control their salt intake than those in the lowest Na: K group (61.5\%, $n=15$ vs $42.7 \%, n=166, p<0.01$ ) (Table 3).

Excluding all participants on treatment for hypertension $(n=154)$, a Spearman's rank order correlation showed no association between $\mathrm{Na}$ excretion, $\mathrm{K}$ excretion or Na: K ratio and SBP, DBP or PP. We also found no significant difference between the regression slopes of SBP, DBP or PP with age in low $(<5 \mathrm{~g} / \mathrm{d}, n=154)$, medium $(5-9 \mathrm{~g} / \mathrm{d}, n=220)$ and high $(>9 \mathrm{~g} / \mathrm{d}, n=302)$ salt groups (Fig. 2). However, there was an association between $\mathrm{BP}$ and the age regression slope according to urinary Na: K ratio. Compared with low Na: K ratio $(<2)$ and medium Na: K (2-5) ratios, the high Na: K group $(>5)$ had a significantly steeper slope with age for both SBP and DBP ( $p=0.01$ and $p<0.01$ respectively). No significant differences for the slopes in all three Na: K groups was found for PP (Fig. 3). Among the high salt group $(>9 \mathrm{~g} / \mathrm{d})$, univariate analysis showed a significant relationship between age, father's education, diabetes, $\mathrm{K}$ and physical activity with SBP while sex, age and father's education were associated with DBP.

\section{Discussion}

In a nationally representative sample of adult Ghanaians ( 50 years and older), we report that $24 \mathrm{~h}$ urinary $\mathrm{Na}$ excretion equated to a median salt intake of $8.3 \mathrm{~g} /$ day, with more than three-quarters of participants having salt intakes in excess of the WHO recommendation of $<5 \mathrm{~g} /$ day. This is considerably higher than median salt consumption values of $6.8 \mathrm{~g}$ and $7.2 \mathrm{~g} /$ day reported by recent studies from South Africa [31, 39], a country that has adopted 
Table 3 Nested cohort by urinary sodium to potassium (Na:K) ratio, low, medium and high groups, WHO - SAGE Ghana Wave 3 (2019)

\begin{tabular}{|c|c|c|c|c|}
\hline Characteristics & $\begin{array}{l}\text { Low Na: } \mathrm{K}(<2) \\
n=399\end{array}$ & $\begin{array}{l}\text { Medium } \\
\text { Na: K (2-5) } \\
n=389\end{array}$ & $\begin{array}{l}\text { High Na: K (>5) } \\
n=41\end{array}$ & $p$ value \\
\hline Age yrs & $60(18)$ & $61(19)$ & $58(22)$ & 0.44 \\
\hline Sex male, $n(\%)$ & $\begin{array}{l}n=399 \\
139(34.8)\end{array}$ & $\begin{array}{l}n=389 \\
116(29.8)\end{array}$ & $\begin{array}{l}n=41 \\
12(29.3)\end{array}$ & 0.29 \\
\hline Ethnicity n (\%) & $n=397$ & $n=387$ & $n=41$ & $<0.01$ \\
\hline Akan & $229(57.7)$ & $246(63.6)$ & $17(41.5)$ & \\
\hline Ga & $5(1.3)$ & $29(7.5)$ & $10(24.4)$ & \\
\hline Ewe & $31(7.8)$ & $23(5.9)$ & $1(2.4)$ & \\
\hline Mole-dagbon & $13(3.3)$ & $11(2.8)$ & $0(0.0)$ & \\
\hline Mole-busanga & $2(0.5)$ & $1(0.3)$ & $0(0.0)$ & \\
\hline Grusi & $7(3.0)$ & $5(1.3)$ & $0(0.0)$ & \\
\hline Guan & $12(3.0)$ & $4(1.0)$ & $0(0.0)$ & \\
\hline Gruma & $3(0.8)$ & $3(0.8)$ & $0(0.0)$ & \\
\hline Other & $95(23.9)$ & $65(16.8)$ & $173(21)$ & \\
\hline Urban n (\%) & $\begin{array}{l}n=398 \\
183(46.0)\end{array}$ & $\begin{array}{l}n=389 \\
240(61.7)\end{array}$ & $\begin{array}{l}n=41 \\
26(63.4)\end{array}$ & $<0.01$ \\
\hline Education (years) & $\begin{array}{l}n=253 \\
10(4)\end{array}$ & $\begin{array}{l}n=267 \\
10(5)\end{array}$ & $\begin{array}{l}n=26 \\
10(6)\end{array}$ & 0.51 \\
\hline$B M I \mathrm{~kg} / \mathrm{m}^{2}$ & $\begin{array}{l}n=387 \\
24.8(7.0)\end{array}$ & $\begin{array}{l}n=364 \\
25.7(7.4)\end{array}$ & $\begin{array}{l}n=39 \\
28.1(8.2)\end{array}$ & 0.04 \\
\hline Salt intake g/d & $\begin{array}{l}n=398 \\
7.5(7.4)^{\mathrm{ab}}\end{array}$ & $\begin{array}{l}n=384 \\
8.6(6.6)^{\mathrm{ac}}\end{array}$ & $\begin{array}{l}n=40 \\
13.9(11.9)^{b c}\end{array}$ & $<0.01$ \\
\hline K intake, $\mathrm{mmol} / \mathrm{d}$ & $\begin{array}{l}n=399 \\
93.4(97.8)^{\mathrm{ab}}\end{array}$ & $\begin{array}{l}n=389 \\
49.5(38.7)^{b}\end{array}$ & $\begin{array}{l}n=41 \\
38.2(38.4)^{\mathrm{a}}\end{array}$ & $<0.01$ \\
\hline $\mathrm{Na}: \mathrm{K}$ & $\begin{array}{l}n=399 \\
1.4(0.6)^{\mathrm{ab}}\end{array}$ & $\begin{array}{l}n=389 \\
3.0(1.3)^{\mathrm{ac}}\end{array}$ & $\begin{array}{l}n=41 \\
5.5(1.3)^{b c}\end{array}$ & $<0.01$ \\
\hline Urinary iodine excretion, mmol/day & $\begin{array}{l}n=383 \\
129.7(116.0)^{a}\end{array}$ & $\begin{array}{l}n=370 \\
153.9(164.4)\end{array}$ & $\begin{array}{l}n=40 \\
146.9(193.9)^{a}\end{array}$ & $<0.01$ \\
\hline Systolic $B P, \mathrm{mmHg}$ & $\begin{array}{l}n=398 \\
126(26)\end{array}$ & $\begin{array}{l}n=385 \\
126(32.0)\end{array}$ & $\begin{array}{l}n=40 \\
125(34.0)\end{array}$ & 0.03 \\
\hline Diastolic BP, $\mathrm{mmHg}$ & $\begin{array}{l}n=398 \\
77(17.0)\end{array}$ & $\begin{array}{l}n=385 \\
75(17.0)\end{array}$ & $\begin{array}{l}n=40 \\
77(18.0)\end{array}$ & 0.25 \\
\hline Pulse pressure, $\mathrm{mmHg}$ & $\begin{array}{l}n=398 \\
48(16.6)\end{array}$ & $\begin{array}{l}n=385 \\
51(17.0)\end{array}$ & $\begin{array}{l}n=40 \\
51.7(15.0)\end{array}$ & 0.26 \\
\hline Mean Arterial Pressure (MAP), $\mathrm{mmHg}$ & $\begin{array}{l}n=399 \\
93.7(18.8)\end{array}$ & $\begin{array}{l}n=387 \\
92.5(21.2)\end{array}$ & $\begin{array}{l}n=41 \\
91.7(25.3)\end{array}$ & 0.61 \\
\hline Hypertension Prevalence, n (\%) & $\begin{array}{l}n=398 \\
170(42.7)\end{array}$ & $\begin{array}{l}n=387 \\
178(46.0)\end{array}$ & $\begin{array}{l}n=40 \\
18(45.0)\end{array}$ & 0.65 \\
\hline Hypertension awareness, $\mathrm{n}$ (\% of hypertension prevalence) & $\begin{array}{l}n=170 \\
102(60.0)\end{array}$ & $\begin{array}{l}n=178 \\
106(59.6)\end{array}$ & $\begin{array}{l}n=18 \\
11(61.1)\end{array}$ & 0.10 \\
\hline $\mathrm{AHT}$, current use $\mathrm{n}$ (\% of hypertension awareness) & $\begin{array}{l}n=102 \\
65(63.7)\end{array}$ & $\begin{array}{l}n=106 \\
79(74.8)\end{array}$ & $\begin{array}{l}n=11 \\
7(63.6)\end{array}$ & 0.22 \\
\hline Hypertension control, n (\% of AHT) & $\begin{array}{l}n=63 \\
33(52.5)\end{array}$ & $\begin{array}{l}n=78 \\
40(51.3)\end{array}$ & $\begin{array}{l}n=7 \\
3(42.9)\end{array}$ & 0.89 \\
\hline Stroke, n (\%) & $\begin{array}{l}n=399 \\
11(2.8)\end{array}$ & $\begin{array}{l}n=389 \\
7(1.8)\end{array}$ & $\begin{array}{l}n=41 \\
0(0.0)\end{array}$ & 0.45 \\
\hline Diabetes, n (\%) & $\begin{array}{l}n=399 \\
34(8.5)\end{array}$ & $\begin{array}{l}n=389 \\
30(7.7)\end{array}$ & $\begin{array}{l}n=41 \\
2(4.9)\end{array}$ & 0.69 \\
\hline Tobacco use, $n(\%)$ & $\begin{array}{l}n=399 \\
45(11.3)\end{array}$ & $\begin{array}{l}n=389 \\
34(8.7)\end{array}$ & $\begin{array}{l}n=41 \\
0(0.0)\end{array}$ & 0.05 \\
\hline Alcohol use, $n(\%)$ & $\begin{array}{l}n=399 \\
160(40.1)\end{array}$ & $\begin{array}{l}n=389 \\
172(44.2)\end{array}$ & $\begin{array}{l}n=41 \\
19(46.3)\end{array}$ & 0.44 \\
\hline
\end{tabular}


Table 3 Nested cohort by urinary sodium to potassium (Na:K) ratio, low, medium and high groups, WHO - SAGE Ghana Wave 3 (2019) (Continued)

\begin{tabular}{|c|c|c|c|c|}
\hline Characteristics & $\begin{array}{l}\text { Low Na: } \mathrm{K}(<2) \\
n=399\end{array}$ & $\begin{array}{l}\text { Medium } \\
\text { Na: K (2-5) } \\
n=389\end{array}$ & $\begin{array}{l}\text { High Na: K (>5) } \\
n=41\end{array}$ & $p$ value \\
\hline Fruits and Veg., met recommendation $n(\%)$ & $\begin{array}{l}n=399 \\
212(53.1)\end{array}$ & $\begin{array}{l}n=389 \\
203(52.2)\end{array}$ & $\begin{array}{l}n=41 \\
24(58.5)\end{array}$ & 0.74 \\
\hline Frequently add salt to food at the table, $n(\%)$ & $\begin{array}{l}n=399 \\
52(13)\end{array}$ & $\begin{array}{l}n=389 \\
48(12.3)\end{array}$ & $\begin{array}{l}n=41 \\
6(14.6)\end{array}$ & 0.88 \\
\hline Frequently add salt to cooking at home, $n(\%)$ & $\begin{array}{l}n=399 \\
320(80.0)\end{array}$ & $\begin{array}{l}n=389 \\
311(79.9)\end{array}$ & $\begin{array}{l}n=41 \\
33(80.5)\end{array}$ & 0.30 \\
\hline Believes that they are eating just the right amount of salt $n(\%)$ & $\begin{array}{l}n=397 \\
247(62.2)\end{array}$ & $\begin{array}{l}n=387 \\
231(59.7)\end{array}$ & $\begin{array}{l}n=41 \\
24(58.5)\end{array}$ & 0.48 \\
\hline Believes a high salt diet can cause a serious health problem, $n(\%)$ & $\begin{array}{l}n=389 \\
304(78.1)\end{array}$ & $\begin{array}{l}n=375 \\
311(82.9)\end{array}$ & $\begin{array}{l}n=40 \\
30(75.0)\end{array}$ & 0.18 \\
\hline Does something to control salt consumption, $n(\%)$ & $\begin{array}{l}n=389 \\
166(42.7)\end{array}$ & $\begin{array}{l}n=375 \\
209(55.7)\end{array}$ & $\begin{array}{l}n=39 \\
15(61.5)\end{array}$ & $<0.01$ \\
\hline
\end{tabular}

Data are presented as median (IQR, interquartile range) unless otherwise stated. BMI, body; hypertension prevalence, $\mathrm{BP} \geq 140 / 90$ or previous diagnosis; $\mathrm{AHT}$, antihypertensive medication use in the last 2 weeks; BMI, body mass index; tobacco use by self-report; alcohol use by self-report; met fruits and vegetable recommendation indicates consumed five or more servings of fruits and vegetables a day; salt behaviour responses, frequently indicates 'always' and 'often'; continuous variables compared using Independent Kruskal-Wallis Test and categorical variables compared using Pearson ChiSquare Test and Fisher's Exact Test. a, b, c; sig at $p<0.05$

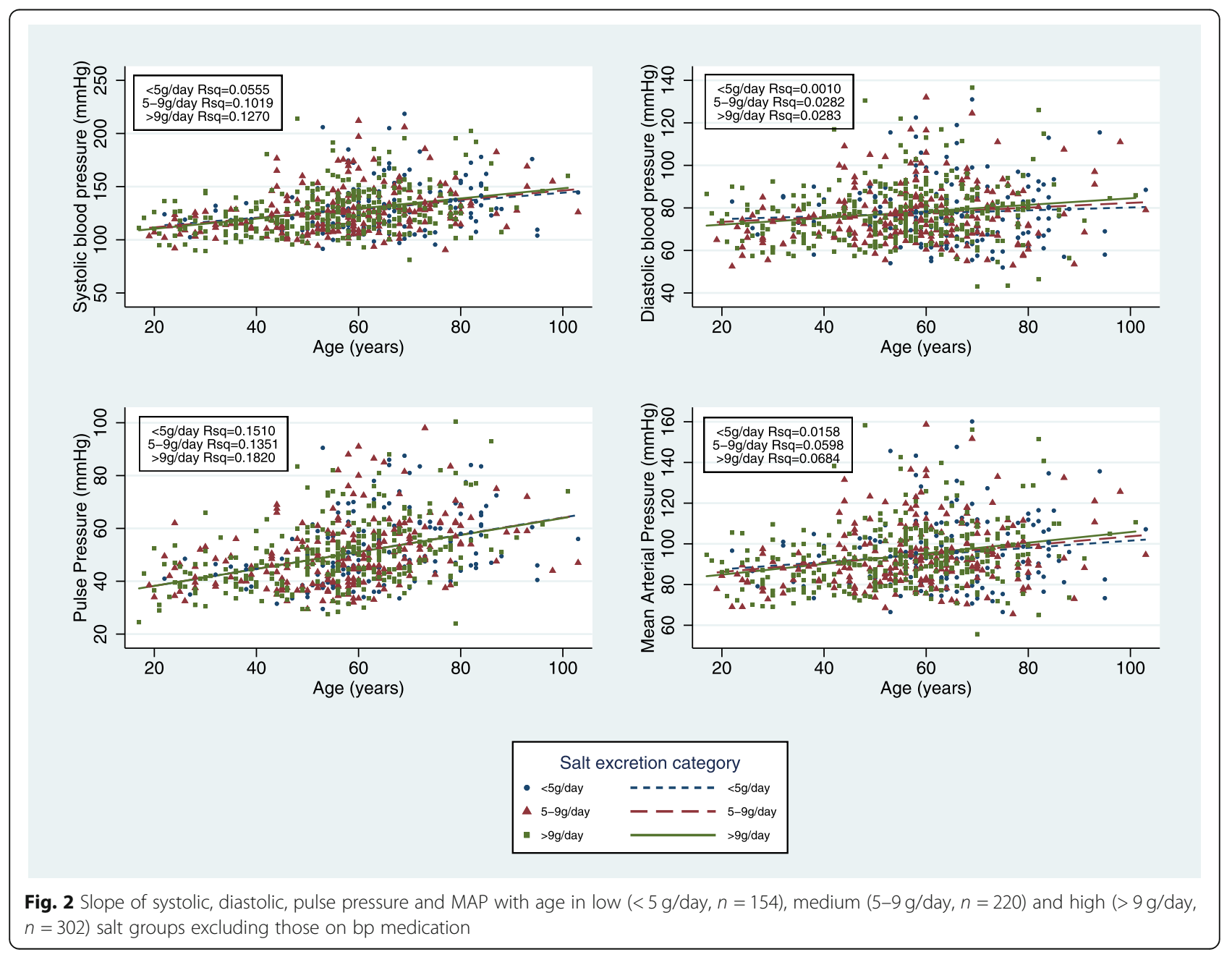



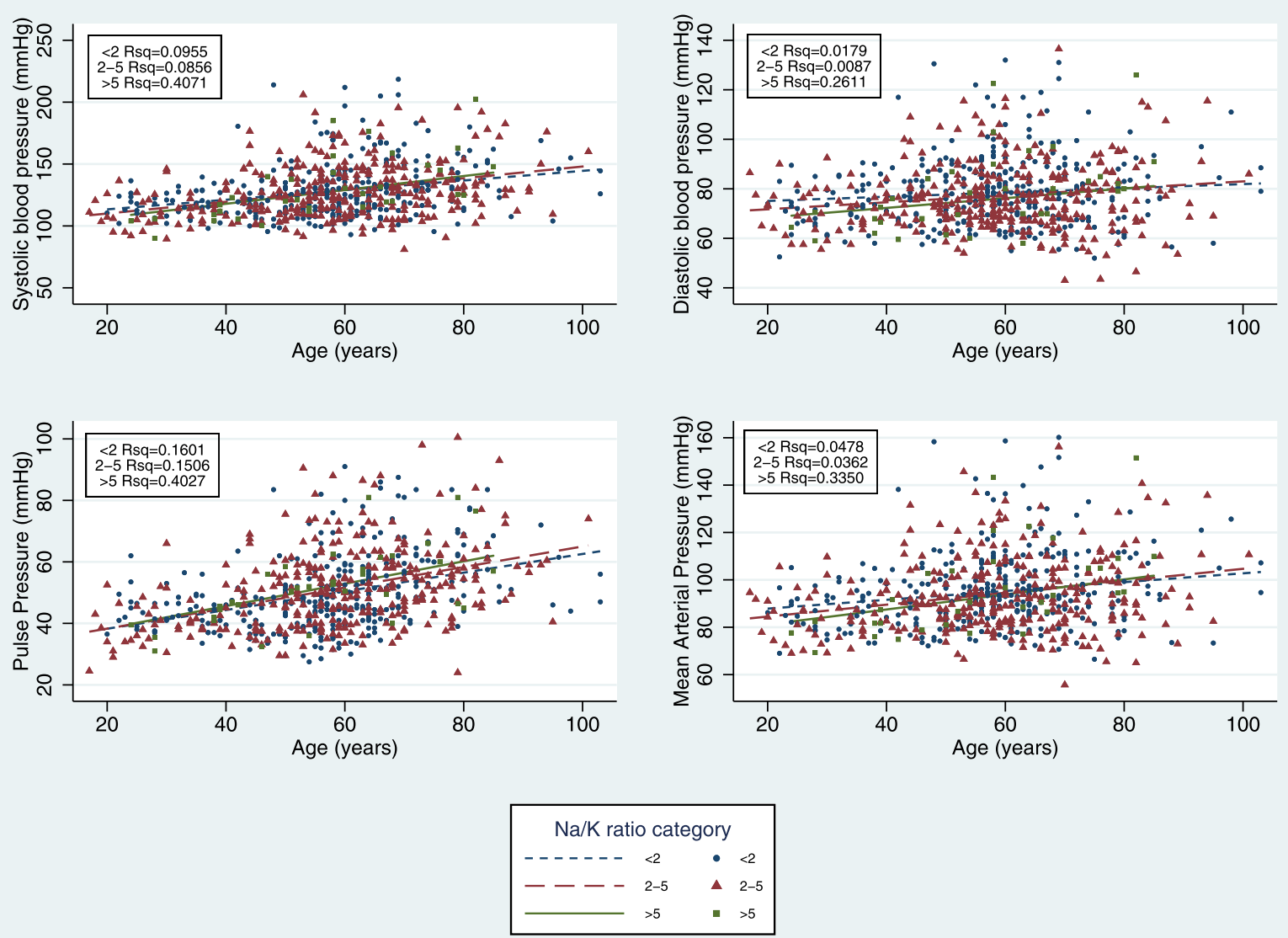

Fig. 3 Slope of systolic, diastolic and pulse pressure with age in low $(<2 \mathrm{mmol} / \mathrm{mmol}, n=334)$, medium ( $2-5 \mathrm{mmol} / \mathrm{mmol}, n=308)$ and high $(>$ $5 \mathrm{mmol} / \mathrm{mmol}, n=34) \mathrm{Na}$ : K groups excluding those on bp medication

mandatory salt reduction policies. Surprisingly, salt intake in the current study was higher in women than men, which is in contrast to findings of other studies [40, 41], and warranting further investigation.

Our data provides much-needed information required to inform strategies to meet the WHO global voluntary target for NCD prevention of 30\% reduction in mean population intake of salt, with the aim of achieving a target of less than $5 \mathrm{~g}$ per day by 2025 [8]. The only other available information on $24 \mathrm{~h}$ urinary $\mathrm{Na}$ excretion in Ghana was collected over a decade ago when salt intake was estimated to be $6.0 \mathrm{~g} /$ day [42]. Over this time, the Ghanaian food environment has changed dramatically $[43,44]$ with the emergence of energy dense, nutrient poor, processed convenience foods such as instant noodles, salty snacks (eg, potato, corn, and tortilla chips), visible fat (e.g. butter, margarine, oils, dressings and gravies), ready-made baked foods (e.g. cookies, cakes, pies and pastries), desserts such as puddings and cheesecake, and sweetened products (e.g. sugar, syrup, ice cream, candy, and carbonated and noncarbonated sweetened drinks). Additionally, frequent consumption of highly salted foods, such as fish and meat, remains part of the traditional Ghanaian cuisine, and salt use in cooking remains high $[42,45]$.

It is noteworthy that more than three quarters of participants in the current study reported adding salt to food during cooking. Poor salt use behavior is widespread in the Ghanaian population; a previous study involving 12 villages in Ghana in 2006 reported that 98\% of participants added salt to food during cooking, while about half (52\%) added salt to food at the table [21]. In comparison to South Africans, Ghanaians reported adding salt to food during cooking more frequently [45], presumably because mass media public health campaigns to lower salt use, such as Salt Watch in South Africa have not yet occurred in Ghana [46]. Further, Ghana's national salt iodization programme, along with accompanying major public health campaigns on iodine consumption [47-50] may partially be responsible for an increase in discretionary salt intake. However, lack of information on sources of salt provided from discretionary and non-discretionary 
sources, as in processed foods, prevents further hypothesis in this regard.

Despite the current study not including a dietary assessment component, a recent systematic review of dietary sources of salt in LMICs identified that bread, meat and meat products, bakery products, instant noodles, salted preserved foods, milk and dairy products, and condiments were major sources thereof [51]. In some LMICs, bread alone may contain as much as $1.36 \mathrm{~g}$ salt per $100 \mathrm{~g}$ bread [52]. For many African countries, bread has become a staple food, such as in some regions of Democratic Republic of Congo, where bread has replaced cassava [53]. The nutrition transition taking place in Sub Saharan Africa and its transformation of dietary patterns from a reliance on traditional staples to an increased intake of energy dense, nutrient poor foods (and often $\mathrm{Na}$ rich) foods may partly be responsible for the observed high salt intake reported by this study. In support of this, the Ghana Demographic and Health Survey 2014 reported that more than a third (36\%) of participants consumed salted dried fish [54], while in the Ghana National Iodine Survey Report 2015, consumption of bouillon cubes was frequent and widespread with nearly half (48.8\%) of the participants having consumed this item at least 6 times a week [55].

Awareness of excessive salt intake was low in the current study. Sixty one percent of participants believed that they were meeting the salt recommendation guidelines, but only $22.3 \%$ actually achieved this. Estimations of dietary salt intake using dietary recall methods usually indicates a reduced daily intake compared to $24 \mathrm{~h}$ urinary collections [56-58]. The tendency to under-estimate salt consumption is high as many people are unaware of the recommendation for salt consumption, unable to determine the salt content of everyday foods such as bread and cereals, or that of composite meals [59-61]. In Ghana, there are no salt reduction strategies in place such as: mandatory or voluntary levels of salt permitted in processed foods; regulations for food labelling to indicate salt content in products, or front-of-pack signposting for high salt warnings. In addition, the Ghanaian food composition tables are outdated and incomplete in the case of $\mathrm{Na}$ [62], making it difficult to assess food contributors to total salt intake. In view of this, regular monitoring and evaluation of the food environment and food consumption patterns are warranted.

Potassium excretion, although low (median $63.8 \mathrm{mmol}$ /day, IQR 74.8, with 65\% not meeting the recommended daily intake of $\mathrm{K}$ ), was higher than that reported by other studies in the region $[31,39]$. The $\mathrm{K}$ content of the food items obtained from the 2010-2011 Ghana Food Balance Sheet indicated that K supply per capita per day was about $9086 \mathrm{mg} /$ day (233 mmol/day), approximately 2.6-fold larger than the WHO recommended level (>90 $\mathrm{mmol} /$ day) with yams, cassava and plantains constituting the bulk of
K supply [63, 64]. However, food balance sheets represent apparent, rather than actual food consumption, and thereby provide an overestimation of intake.

Hypertension prevalence in the current study was high $(50+y, 52.6 \% ; 18-48 y, 14.5 \%)$, with less than two thirds $(60 \%)$ of participants classified as hypertensives being aware of the condition (i.e. diagnosed), of which $69.1 \%$ were being treated with antihypertensive medication, half of which had BP levels considered to be controlled $(<140 / 90 \mathrm{mmHg})$. Surprisingly, we found no association between urinary $\mathrm{Na}, \mathrm{K}$ or $\mathrm{N}: \mathrm{K}$ ratio with $\mathrm{BP}$ in contrasts to findings from other comparable studies [65-67] but in agreement with others [31, 68]. A possible reason may be the day to day variability in salt intake, which results in a high intra-individual variability in urinary $\mathrm{Na}$ excretion. Though $24 \mathrm{~h} \mathrm{Na}$ excretion is considered to be the gold standard method for determination of population $\mathrm{Na}$ intake, repeated $24 \mathrm{~h}$ collections over periods provide more accurate estimating an individual's habitual salt intake $[69,70]$. However, this approach is typically impractical for large population studies such as the one reported here.

Similarly, no difference was found between the slopes of SBP, DBP or PP with age according to three categories of salt intake, namely low (5 g/day), medium (5-9 g/ day) and high (>9g/day). However, high and medium urinary $\mathrm{Na}$ : K had steeper slopes of BP with age as compared with those in the low $\mathrm{Na}$ : K category. A national survey (WHO SAGE Wave 2 South Africa) conducted in 2015 reported similar findings [31]. This association was first explored in the INTERSALT study, the largest multinational study on $24 \mathrm{~h}$ urinary excretion and BP with 10,079 adults across 48 centres worldwide [66]. This implies that a simultaneous increase in $\mathrm{K}$ and a decrease in $\mathrm{Na}$ will be beneficial in reducing $\mathrm{BP}$ with age and ultimately hypertension incidence $[38,66]$. As such, strategies such as taxes on high-salt products, labelling and effective communication, voluntary salt commitments by food industries and legislations for $\mathrm{Na}$ reduction are warranted. Similarly, to increase K intake, nutrition promotion of $\mathrm{K}$ rich fruits and vegetables may be needed, along with policies that make these foods more accessible and affordable.

A strength of the study is the use of a large nationally representative sample of participants aged 50+ years, in keeping with the aim of WHO SAGE studies. This design on the other hand, may limit generalization to the entire population. Another potential limitation is the recruitment strategy which resulted in more women than men. Women are known to be more likely to volunteer to participate in surveys than men [71], and this has been observed in other $24 \mathrm{~h}$ urine collection studies $[31,39]$. Akan is the largest ethnic group in Ghana that is well distributed across the entire country [54] which is 
why this group was proportionally larger than other ethnic groups in the sample. Additionally, the crosssectional analysis limits the ability to evaluate the influence of dietary $\mathrm{Na}$ and $\mathrm{K}$ intake on $\mathrm{BP}$ over time. The choice of urine volumes as a sole determinant of completeness of $24 \mathrm{~h}$ urine collections requires explanation. Presently, there is no universally accepted standard for determining completeness of $24 \mathrm{~h}$ urine collections. The International Consortium for Quality Research on Dietary Sodium/Salt (TRUE), recommends the use of paraaminobenzoic acid (PABA) as being the preferred method [72] but experiences using PABA in African communities is limited and experiences from South Africa [73] suggest that participants may forget or choose not to take their PABA tablets during the urine collection period, thus rendering this method impractical .

The TRUE consortium recognized a need to establish further additional measures of assessing $24 \mathrm{~h}$ urinary completeness beyond PABA. A systemic review found that no single exclusion criteria $(24 \mathrm{~h}$ urinary creatinine excretion, creatinine index $<0.7$, total urinary volume, a combination of creatinine excretion and total urine volume or self-report of missing urine) appeared more accurate in identifying incomplete 24-h urine collections than any others [74]. Importantly, the authors point out that the use of a combination of methods such as creatinine excretion together with urinary volume may lead to the unnecessary exclusion of participants with complete urine samples. The reference values used as a cut-off for inadequate urinary collection based on creatinine values has largely been based on those recommended by Stolarz-Skrzypek et al., namely urinary volume < $300 \mathrm{ml}$, or creatinine excretion $<4 \mathrm{mmol}$ or $>25 \mathrm{mmol}$ for women and $<6 \mathrm{mmol}$ and $>30 \mathrm{mmol}$ for men [28]. The applicability of these creatinine excretion reference values across different ethnic groups has recently been questioned [74]. While the TRUE authors concluded that a creatinine index $<0.7$ may best increase the sensitivity of eliminating incomplete urine sampling, they go on to promote the use of similar methodologies used by the INTERSALT and INTERMAP studies as the preferred method to determine $24 \mathrm{~h}$ urinary completeness in population based studies [27, 75]. These were as follows: samples that fell outside of the collection time of $22-26 \mathrm{~h}$; participants' indication that the collection was incomplete and that they had spilled 'more than a few drops' of urine; or if total urinary volume was $<250 \mathrm{ml}$. Given that creatinine is generally variable and largely dependent on muscle mass and protein intake [74], and the reference cut-off values in African populations have not been widely validated, we opted to use $24 \mathrm{~h}$ urinary volumes $\leq 300 \mathrm{ml}$ as the measure for incomplete collections. The generally low creatinine excretion values found across the entire cohort suggest a possible low dietary protein intake, but a lack of dietary data precludes further consideration in this regard.

\section{Conclusions}

This nationwide study of Ghanaians, using $24 \mathrm{~h}$ urinary collections, reported a high intake of salt, accompanied by a low $\mathrm{K}$ intake. Increasing urinary $\mathrm{Na}$ : $\mathrm{K}$ ratio was associated with increasing BP with age. Given the high prevalence of hypertension in the study, our findings identify a need for population-wide strategies to reduce dietary salt intake whilst, at the same time, increase $\mathrm{K}$ intake in Ghana.

\section{Supplementary information}

Supplementary information accompanies this paper at https://doi.org/10. 1186/s40795-020-00379-y.

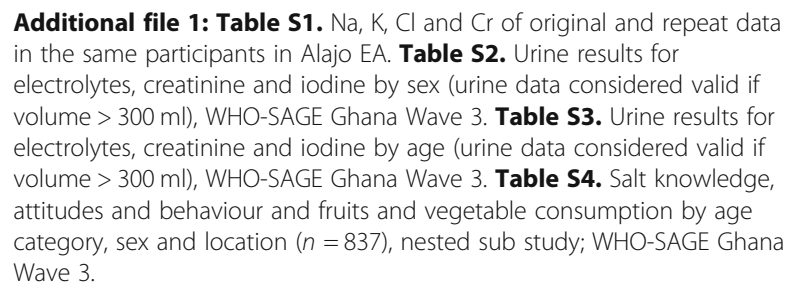

\section{Abbreviations}

CVDs: Cardiovascular diseases; Na: Sodium; K: Potassium; Cr: Creatinine; LMICs: Low- and Middle-Income Countries; NCDs: Non communicable diseases; WHO: World Health Organization; SHAKE: Surveillance, Harness industry, Adopt Standards for marketing and labelling, Knowledge and Environment; BP: Blood Pressure; SAGE: Study on global AGEing and adult health; CAPI: Computer Assisted Personal Interview; PAHO: Pan American Health Organization; NMIMR-UG: Noguchi Memorial Institute for Medical Research of the University of the Ghana; INTERMAP: International Population Study on Macronutrients and Blood Pressure; INTERSALT: International Study of Sodium, Potassium, and Blood Pressure; EAs: Enumerator Areas;

SBP: Systolic Blood Pressure; DPB: Diastolic Blood Pressure; PP: Pulse Pressure; MAP: Mean Arterial Pressure; IQR: Inter Quartile Range; BMI: Body Mass Index; TRUE: The International Consortium for Quality Research on Dietary Sodium/ Salt; PABA: Para-aminobenzoic acid

\section{Acknowledgements \\ The authors thank all participants for their contributions. We acknowledge the support of Noguchi Memorial Institute for Medical Research of the University of the Ghana and Liberty Medical Laboratory, Accra for the analysis of urinary electrolytes.}

\section{Disclaimer}

The content of this manuscript is solely the responsibility of the authors and does not necessarily represent the official views of the World Health Organization or the funding bodies.

\section{Authors' contributions}

Authors contributions were as follows: KC, PK and LW designed the study; RB implemented the research; NM, BC and EM managed the data and conducted the analysis; EM, KC, JR, AES wrote the paper. KC takes responsibility of the content of this paper. All authors read and approved of the final manuscript. 


\section{Funding}

This work is supported by an agreement with the CDC Foundation with financial support provided by Bloomberg Philanthropies, and a Partnerships \& Research Development Fund (PRDF) grant from the Australia Africa Universities Network. SAGE is supported by WHO and the Division of Behavioral and Social Research (BSR) at the National Institute on Aging (NIA), US National Institutes of Health, through Interagency Agreements with WHO [OGHA 04034785; YA1323-08-CN-0020; Y1-AG-1005-01] and a Research Project Grant [R01AG034479]. LW is supported by the South African DSI-NRF Centre of Excellence in Human Development.

\section{Availability of data and materials}

The datasets used and/or analyzed during the current study are available from the corresponding author on reasonable request.

\section{Ethics approval and consent to participate}

The study obtained approval from the WHO Ethics Committee (RPC 149) and the University of Ghana Medical School Ethics and Protocol Review Committee (MS-Et/M.03 - P 3.1/2005-2006). Participants provided written informed consent following explanation of the study procedure in their home language.

\section{Consent for publication}

Not applicable.

\section{Competing interests}

The authors declare that they have no competing interests.

\section{Author details}

${ }^{1}$ School of Medicine, Faculty of Science, Medicine and Health, University of Wollongong, Northfields Avenue, Wollongong, NSW 2522, Australia. ${ }^{2}$ Neuroscience Institute, National Research Council, Padova, Italy. ${ }^{3}$ School of Health and Society, Faculty of Social Sciences, University of Wollongong, Northfields Avenue, Wollongong, NSW 2522, Australia. ${ }^{4}$ SAMRC/Wits Developmental Pathways for Health Research Unit, University of the Witwatersrand, Johannesburg, South Africa. ${ }^{5}$ DSI-NRF Centre of Excellence in Human Development, University of the Witwatersrand, Johannesburg, South Africa. ${ }^{6}$ Department of Community Health, University of Ghana, Accra, Ghana. ${ }^{7}$ Chiang Mai University Research Institute for Health Sciences, Chiang Mai, Thailand. ${ }^{8}$ World Health Organization (WHO), Geneva, Switzerland. ${ }^{9}$ School of Public Health and Community Medicine, University of New South Wales; The George Institute for Global Health, Sydney, NSW 2052, Australia.

${ }^{10}$ Hypertension in Africa Research Team, North-West University, Potchefstroom 2520, South Africa. ${ }^{11}$ Illawarra Health and Medical Research Institute, Wollongong, NSW 2522, Australia.

Received: 4 May 2020 Accepted: 4 September 2020 Published online: 29 September 2020

\section{References}

1. Cardiovascular diseases (CVDs) https://www.who.int/newsroom/factsheets/detail/cardiovasculardiseases(cvds). Accessed 12 Jul 2019.

2. WHO. Global status report on noncommunicable diseases 2010. Geneva: WHO; 2011.

3. Erratum. A comparative risk assessment of burden of disease and injury attributable to 67 risk factors and risk factor clusters in 21 regions, 19902010: a systematic analysis for the Global Burden of Disease Study 2010. Lancet. 2013;381(9874):1276.

4. Kearney PM, Whelton M, Reynolds K, Muntner P, Whelton PK, He J. Global burden of hypertension: analysis of worldwide data. Lancet. 2005;365(9455): 217-23.

5. Hypertension. https://www.who.int/newsroom/factsheets/detail/ hypertension. Accessed 07 Aug 2019.

6. Centre for Health Information Management. Outpatient morbidity in health facilities, Ghana. Accra: Ghana Health Service; 2008.

7. Sanuade O, Boatemaa S, Kushitor M. Hypertension prevalence, awareness, treatment and control in Ghanaian population: Evidence from the Ghana demographic and health survey. PLoS ONE. 2018;13(11):e0205985. https:// doi.org/10.1371/journal.pone.0205985.
8. WHO: About 9 voluntary global targets. Global Monitoring Framework for NCDs. https://www.who.int/nmh/ncd-tools/definition-targets/en/. Accesed 22 Feb 2020.

9. WHO and World Economic Forum. From burden to "best buys": reducing the economic impact of non-communicable diseases in low- and middleincome countries. Geneva: World Economic Forum; 2011.

10. Salt Reduction. https://www.who.int/news-room/fact-sheets/detail/saltreduction. Accessed 06 Aug 2019.

11. Institute of Medicine (US). Panel on Dietary Reference Intakes for Electrolytes and Water. In: DRI, dietary reference intakes for water, potassium, sodium, chloride, and sulfate. Washington, DC: National Academies Press; 2005.

12. He FJ. How far should salt intake be reduced? Hypertension. 2003;42(6): 1093-9.

13. He FJ, Li J, Macgregor GA. Effect of longer-term modest salt reduction on blood pressure. Cochrane Database Syst Rev. 2013;346:f1325325. https://doi. org/10.1136/bmj.f1325.

14. Strazzullo P, D'Elia L, Kandala NB, Cappuccio FP. Salt intake, stroke, and cardiovascular disease: Meta-analysis of prospective studies. BMJ (Online). 2009;339(7733):1296

15. Graudal NA, Hubeck-Graudal T, Jurgens $G$. Effects of low sodium diet versus high sodium diet on blood pressure, renin, aldosterone, catecholamines, cholesterol, and triglyceride. Cochrane Database Syst Rev. 2017;2017(4).

16. Dickinson HO, Mason JM, Nicolson DJ, Campbell F, Beyer FR, Cook JV, et al. Lifestyle interventions to reduce raised blood pressure: a systematic review of randomized controlled trials. J Hypertens. 2006;24(2):215-23.

17. Dietary Guidelines Advisory Committee. Adults and sodium: what is the relationship between sodium and blood pressure in adults aged 19 years and older? Washington, D.C: Department of Health and Human Services and Department of Agriculture; 2010.

18. Cobb LK, Anderson CAM, Elliott P, Hu FB, Liu K, Neaton JD, et al. Methodological issues in cohort studies that relate sodium intake to cardiovascular disease outcomes: a science advisory from the american heart association. Circulation. 2014;129(10):1173-86.

19. WHO. 'SHAKE' the salt habit. Geneva: WHO; 2016.

20. Sookram CDM, Phori PM, Varenne B, Alisalad A. WHO's supported interventions on salt intake reduction in the sub-Saharan Africa region. Cardiovasc Diagn Ther. 2015;5(3):186-90.

21. Cappuccio FP, Kerry SM, Micah FB, Plange-Rhule J, Eastwood JB. A community programme to reduce salt intake and blood pressure in Ghana [ISRCTN88789643]. BMC Public Health. 2006;6.

22. Chockalingam A, Balaguer-Vintro I, Achutti A, De Luna AB, Chalmers J, Farinaro $E$, et al. The world heart federation's white book: impending global pandemic of cardiovascular diseases: challenges and opportunities for the prevention and control of cardiovascular diseases in developing countries and economies in transition. Can J Cardiol. 2000;16(2):227-9.

23. Charlton K, Ware L, Menyanu E, Biritwum RB, Naidoo N, Pieterse C, et al. Leveraging ongoing research to evaluate the health impacts of South Africa's salt reduction strategy: A prospective nested cohort within the WHO-SAGE multicountry, longitudinal study. BMJ Open. 2016;6(11).

24. Kowal P, Chatterji S, Naidoo N, Biritwum R, Fan W, Ridaura RL, et al. Data resource profile: the World Health Organization study on global AGEing and adult health (SAGE). Int J Epidemiol. 2012;41:1639-49.

25. World Health Organization. National Report of the World Health Survey in Ghana, 2002-2004. Geneva: WHO; 2006.

26. Protocol for population level sodium determination in $24 \mathrm{hr}$ urine samples. https://www.paho.org/hq/dmdocuments/2013/24h-urine-Protocol-eng.pdf. Accessed 15 Jan 2020

27. Elliott P. Sodium intakes around the world. Background document prepared for the forum and technical meeting on reducing salt intake in populations (Paris 5-7th October 2006). Geneva: WHO; 2007.

28. Stolarz-Skrzypek K, Kuznetsova T, Thijs L, Tikhonoff V, Seidlerová J, Richart T, et al. Fatal and nonfatal outcomes, incidence of hypertension, and blood pressure changes in relation to urinary sodium excretion. J Am Med Assoc. 2011;305(17):1777-85.

29. Ohashi T, Yamaki M, Pandav CS, Karmarkar MG, Irie M. Simple microplate method for determination of urinary iodine. Clin Chem. 2000;46(4):529-36.

30. Ribeirol KCB, Nolasco EL, Vanellil CP, Mesquita HL, Corrêa JOA. Urine storage under refrigeration preserves the sample in chemical, cellularity and bacteriuria analysis of ACS. J Bras Patol Med Lab. 2013;49(6). 
31. Ware LJ, Charlton K, Schutte AE, Cockeran M, Naidoo N, Kowal P. Associations between dietary salt, potassium and blood pressure in south African adults: WHO SAGE wave 2 Salt \& Tobacco. Nutr Metab Cardiovasc Dis. 2017;27(9):784-91.

32. Topouchian JA, El Assaad MA, Orobinskaia LV, El Feghali RN, Asmar RG. Validation of two automatic devices for self-measurement of blood pressure according to the international protocol of the European Society of Hypertension: the Omron M6 (HEM-7001-E) and the Omron R7 (HEM 637IT). Blood Pressure Monitoring. 2006;11(3):165-71.

33. O'Brien E, Pickering T, Asmar R, Myers M, Parati G, Staessen J, et al. Working group on blood pressure monitoring of the European Society of Hypertension International Protocol for validation of blood pressure measuring devices in adults. Blood Pressure Monitoring. 2002;7(1):3-17.

34. Takahashi Y, Sasaki S, Okubo S, Hayashi M, Tsugane S. Blood pressure change in a free-living population-based dietary modification study in Japan. J Hypertens. 2006;24(3):451-8.

35. Williams B, Mancia G, Spiering W, Rosei EA, Azizi M, Burnier M, et al. 2018 practice guidelines for the management of arterial hypertension of the European society of cardiology and the European society of hypertension ESC/ESH task force for the management of arterial hypertension. J Hypertens. 2018;36(12):2284-309.

36. Selvarag S, Elbez Y, Sorbets E, Feldman LJ, Eagle KA. Pulse pressure and risk for cardiovascular events in patients with atherothrombosis: from the REAC H registry. J Am Coll Cardiol. 2016;67(4):392-403.

37. WHO: Global Physical Activity Questionnaire. https://www.who.int/ncds/surveillance/steps/resources/GPAQ_Analysis_Guide.pdf.

38. Guideline: Potassium intake for adults and children. 2012. http://apps.who. int/iris/bitstream/10665/77986/1/9789241504829_eng.pdf?ua=1\&ua=1 Accessed 21 Aug 2019.

39. Swanepoel B, Schutte AE, Cockeran M, Steyn K, Wentzel-Viljoen E. Sodium and potassium intake in South Africa: an evaluation of 24-hour urine collections in a white, black, and Indian population. J Am Soc Hypertens. 2016;10(11):829-37.

40. Seko C, Odani K, Wada S, Yoshii K, Segawa H, Kitaoka K, et al. Characteristic dietary habits associated with high values of estimated 24-hours urinary sodium excretion and sodium-to-potassium ratio assessed by age group among the residents of a rural town in Japan. Clin Exp Hypertens. 2020; 42(5):449-59.

41. Morrissey E, Giltinan M, Kehoe L, Nugent AP, McNulty BA, Flynn A, et al. J. Sodium and Potassium Intakes and Their Ratio in Adults (18-90 y): Findings from the Irish National Adult Nutrition Survey. Nutrients. 2020;12:938.

42. Kerry SM, Emmett L, Micah FB, Martin-Peprah R, Antwi S, Phillips RO, et al. Rural and semi-urban differences in salt intake, and its dietary sources, in Ashanti, West Africa. Ethn Dis. 2005;15(1):33-9.

43. Monteiro CA, Moubarac JC, Cannon G, Ng SW, Popkin B. Ultra-processed products are becoming dominant in the global food system. Obes Rev. 2013;14(S2):21-8.

44. Andam KS, Tschirley D, Asante SB, Al-Hassan RM, Diao X. The transformation of urban food systems in Ghana: findings from inventories of processed products. Outlook Agric. 2018;47(3):233-43.

45. Menyanu E, Charlton KE, Ware LJ, Russell J, Biritwum R, Kowal P. Salt use behaviours of ghanaians and south africans: A comparative study of knowledge, attitudes and practices. Nutrients. 2017;9(9).

46. Wentzel-Viljoen E, Steyn K, Lombard C, De Villiers A, Charlton K, Frielinghaus $S$, et al. Evaluation of a mass-media campaign to increase the awareness of the need to reduce discretionary salt use in the South African population. Nutrients. 2017;9(11)

47. Ghana: Stakeholder Campaign On the Use of lodated Salt. http://allafrica. com/-stories/-201110060238.html. Accessed 05 May 2020.

48. Intensify Universal Salt lodization Campaign-Official. http://www ghananews-agency.org/print/36. Accessed 04 Apr 2020.

49. ICCIDD. Progress of Household Consumption of lodated Salt in Some African Countries. lodine Defic Disord Newsl. 2008;31:71-8.

50. Combatting IDD in Ghana. http://www.ign.org/newsletter/idd_nl_may06_ ghana.-pdf. Accessed 04 Apr 2020.

51. Menyanu E, Russell J, Charlton K. Dietary Sources of Salt in Low- and Middle-Income Countries: A Systematic Literature Review. Int J Environ Res Public Health. 2019;16(12).
52. Nwanguma BC, Okorie $\mathrm{CH}$. Salt (sodium chloride) content of retail samples of Nigerian white bread: implications for the daily salt intake of normotensive and hypertensive adults. J Hum Nutr Diet. 2013;26(5):488-93.

53. Will Cassava Remain a Staple Food in the Congo? http://www.fao.org/ docrep/-U3550t/. Accessed 8 Mar 2020.

54. Ghana Demographic and Health Survey. http://www.statsghana.gov. gh/docfiles/-publications/2014\%20GDHS\%20\%20Report.pdf. Accessed 10 Mar 2020

55. National lodine Survey Report 2015. https://www.ghanahealthservice.org/downloads/lodine_Survey_Report.pdf Accessed 28 Mar 2020.

56. Keogh JB, Lange K, Hogarth R, Clifton PM. Foods contributing to sodium intake and urinary sodium excretion in a group of Australian women. Public Health Nutr. 2013;16(10):1837-42.

57. Villani AM, Clifton PM, Keogh JB. Sodium intake and excretion in individuals with type 2 diabetes mellitus: a cross-sectional analysis of overweight and obese males and females in Australia. J Hum Nutr Diet. 2012;25(2):129-39.

58. Margerison C, Riddell $\sqcup$, Wattanapenpaiboon N, Nowson CA. Dietary sources and meal distribution of sodium and potassium in a sample of Australian adults. Nutr Diet. 2013;70(4):294-9.

59. Chariton K, Yeatman H, Houweling F, Guenon S. Urinary sodium excretion, dietary sources of sodium intake and knowledge and practices around salt use in a group of healthy Australian women. Aust N Z J Public Health. 2010; 34(4):356-63.

60. Sarmugam R, Worsley A, Flood V. Development and validation of a salt knowledge questionnaire. Public Health Nutr. 2014;17(5):1061-8.

61. Consensus Action on Salt and Health Salt and Your Health-TNS Public Opinion Survey Summary report. http://www.actiononsalt.org.uk/Docs/393 07.pdf. Accessed 07 Mar 2020.

62. Stadlmayr B, Charrondière UR, Burlingame B. Development of a regional food composition table for West Africa. Food Chem. 2013; 140(3):443-6.

63. Food and Agriculture Organization. Food balance sheets: a handbook, Economic and Social Development Department, Food and Agriculture Organization of the United Nations, Rome, Italy; 2012.

64. Yawson DO, Adu MO, Ason B, Armah FA, Boateng E, Quansah R. Ghanaians might be at risk of excess dietary intake of potassium based on food supply data. J Nutr Metab. 2018. https://doi.org/10.1155/2018/5989307.

65. Aburto NJ, Hanson S, Gutierrez H, Hooper L, Elliott P, Cappuccio FP. Effect of increased potassium intake on cardiovascular risk factors and disease: Systematic review and meta-analyses. BMJ (Online). 2013; 346(7903).

66. Rose G, Stamler J, Stamler R, Elliott P, Marmot M, Pyorala K, et al. Intersalt: an international study of electrolyte excretion and blood pressure. Results for 24 hour urinary sodium and potassium excretion. Br Med J. 1988;297(6644): 319-28.

67. Stamler J, Chan Q, Daviglus ML, Dyer AR, Van Horn L, Garside DB, et al. Relation of dietary sodium (salt) to blood pressure and its possible modulation by other dietary factors the intermap study. Hypertension. 2018; 71(4):631-7

68. Charlton KE, Steyn K, Levitt NS, Zulu JV, Jonathan D, Veldman FJ, et al. Diet and blood pressure in South Africa: intake of foods containing sodium, potassium, calcium, and magnesium in three ethnic groups. Nutrition. 2005: 21(1):39-50

69. Olde Engberink RHG, Van Den Hoek TC, Van Noordenne ND, Van Den Born BJH, Peters-Sengers $H$, Vogt L. Use of a single baseline versus multiyear 24-hour urine collection for estimation of long-term sodium intake and associated cardiovascular and renal risk. Circulation. 2017; 136(10):917-26.

70. Lerchl K, Rakova N, Dahlmann A, Rauh M, Goller U, Basner M, et al. Agreement between 24-hour salt ingestion and sodium excretion in a controlled environment. Hypertension. 2015;66(4):850-7.

71. Markanday S, Brennan SL, Gould H, Pasco JA. Sex-differences in reasons for non-participation at recruitment: Geelong Osteoporosis Study. BMC Res Notes. 2013;6(1)

72. Campbell NRC, He FJ, Tan M, Cappuccio FP, Neal B, Woodward M, et al. The International Consortium for Quality Research on Dietary Sodium/Salt (TRUE) position statement on the use of 24-hour, spot, and short duration (\&lt;24 hours) timed urine collections to assess dietary sodium intake. J Clin Hypertens. 2019;21(6):700-9. 
73. Charlton KE, Steyn K, Levitt NS, Peer N, Jonathan D, Gogela T, et al. A foodbased dietary strategy lowers blood pressure in a low socio-economic setting: a randomised study in South Africa. Public Health Nutr. 2008;11(12): 1397-406.

74. John KA, Cogswell ME, Campbell NR, Nowson CA, Legetic B, Hennis AJM, et al. Accuracy and usefulness of select methods for assessing complete collection of 24-hour urine: a systematic review. J Clin Hypertens. 2016;18(5): 456-67.

75. Stamler J, Elliott P, Dennis B, Dyer AR, Kesteloot H, Liu K, et al. INTERMAP: background, aims, design, methods, and descriptive statistics (nondietary). J Hum Hypertens. 2003;17(9):591-608.

\section{Publisher's Note}

Springer Nature remains neutral with regard to jurisdictional claims in published maps and institutional affiliations.

Ready to submit your research? Choose BMC and benefit from:

- fast, convenient online submission

- thorough peer review by experienced researchers in your field

- rapid publication on acceptance

- support for research data, including large and complex data types

- gold Open Access which fosters wider collaboration and increased citations

- maximum visibility for your research: over $100 \mathrm{M}$ website views per year

At BMC, research is always in progress.

Learn more biomedcentral.com/submissions 